\title{
Paper Performance Analysis of SPSK with Dual Polarized Transmit Antennas over Rayleigh Fading Channel
}

\author{
Malarvizhi Subramani, Arumbu Vanmathi Neduncheran, and Vijayakumar Ponnusamy \\ Department of Electronics and Communications Engineering (ECE), SRM IST-Kattankulathur, Chennai, India
}

https://doi.org/10.26636/jtit.2018.118717

\begin{abstract}
In this paper, the Space Polarization Shift Keying (SPSK) system, which is an extended version of Space Shift Keying (SSK) and includes both space and polarization dimensions with dual polarized antennas, is studied. The capacity and error probability analysis of SPSK for different polarization configurations are dealt with over the Rayleigh fading channel with a rich scattering environment. The analysis conducted shows that the SPSK with a dual polarized antenna (DP) offers better results than SPSK with a single orthogonal polarized antenna SPSK(SP), under non-LOS conditions. SPSK(DP) outperforms SSK by $4.2 \mathrm{~dB}$ over the correlated Rayleigh fading channel at $3 \mathrm{bits} / \mathrm{s} / \mathrm{Hz}$.
\end{abstract}

Keywords-ABER, dual polarized antenna, Rayleigh fading, spatial modulation, SPSK, SSK.

\section{Introduction}

In wireless communications, the need for high data exchange rates and spectral efficiency is increasing on a continuous basis. Demand for spectral efficiency means that the power required for transmission is growing as well. As the power increases, more radiation will be emitted from the antenna which, in turn, increases the pollution. So, reduction of the transmit power, achieved with a simultaneous data rate increase, is the major concern. High data exchange and spectral efficiency rates can be achieved by using more antennas, both in the transmitter and the receiver, and this means that the Multiple Input Multiple Output (MIMO) scheme is employed. The higher number of transmit antennas increases the data exchange rate and information is transmitted in a parallel way.

One such case is the Vertical-Bell Laboratories Layered Space- Time system (V-BLAST), where information is spilt into many parallel sub-streams which are allowed to transmit from all transmit antennas at the same time. This increases both the data rate and the spectral efficiency, but synchronization is required for all the transmit antennas to send information at the same time. V-BLAST employs spatial multiplexing here. The major disadvantage is linked to Inter Channel Interference (ICI) and Inter Antenna Interference (IAI). As more antennas are active at the same time, many RF channels are required to transmit the information, which increases the level of radiation.
In the development of green wireless communication spatial modulation (SM), as introduced by Mesleh et al., where multiplexing was used to increase the data rate of a single antenna system, it is proposed that the complexity of the receiver design should remain low [1]. In SM, only one antenna is active at a given time, hence one RF chain is required which, in turn, reduces radiation. IAI and ICI can be avoided. In SM information is conveyed through both modulation index $M$ and antenna index $N_{t}$. As the number of transmit antennas increases, the number of information bits increases as well. The total number of information bits transmitted is $\log _{2}\left(M \times N_{t}\right)$. In Space Shift Keying (SSK), however, the information is conveyed through the antenna index only [2].

Polarization Shift Keying Modulation (PSKM) is presented in [3], where binary bits are encoded as orthogonal states of polarization. In contrast to binary PSKM scheme, the multi-level PSKM scheme supports higher data rates, as multiple bits are encoded in one symbol. An additional polarization index is also added to the antenna index to relay the information, known as Space Polarization Shift Keying (SPSK) [4]. In SPSK, instead of a modulation index, a polarization index $P$ is used along with dual polarized antennas at both input and output points. So, the total number of bits transmitted is $\log _{2}\left(2 N_{t} \times P\right)$. For example, instead of using four single polarized transmit antennas in SM, only two dual polarized antennas are used here, which reduces the installation cost and also improves the spectral efficiency compared to single polarized antennas. Applications like Internet of Things and mobile devices with receive antenna orientation may opt for SPSK(DP) to improve spectral efficiency.

This paper is organized as follows: Section 2 introduces the system model. Section 3 deals with the performance analysis in detail. Section 4 discusses the results achieved by the system proposed in this paper.

\section{System Model}

A general system model is shown in Fig. 1, consisting of $N_{t}$ dual polarized transmit antennas and $N_{r}$ dual polarized receive antennas. A random sequence of information bits 


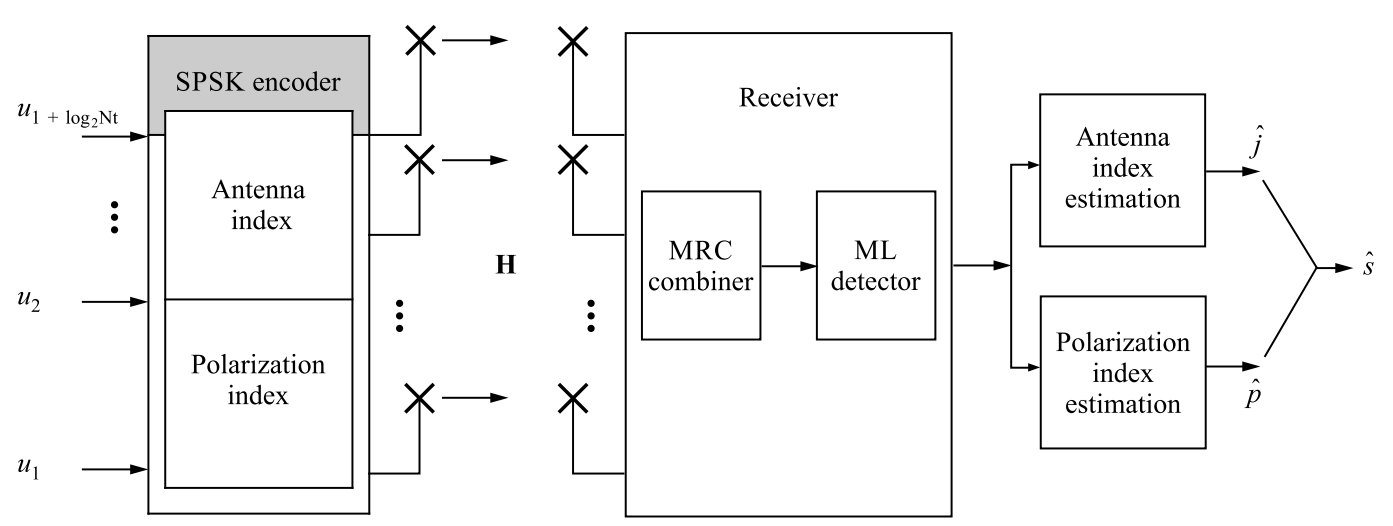

Fig. 1. SPSK system model with dual polarized antennas.

enters the encoder which groups the information sequence of size $m=2 \log _{2} N_{t}+1$ to a constellation vector:

$s_{j p}=\left\{\begin{array}{ccccccccc}0 & 0 & \ldots & 0 & 1^{p} & 0 & \ldots & 0 & 0 \\ & & & & \uparrow & & & & \\ & & & & j \text {-th position } & & & & \end{array}\right\}$,

where $j$ is the active antenna index and $p$ is the polarization index ( $x$ denotes horizontal and $y$ denotes vertical polarization).

Each group is divided into two blocks, the first bit chooses the polarization index and the next $2 \log _{2} N_{t}$ bits choose the antenna index. In the SPSK system, only one antenna is active during the transmission. All others are idle. Compared to the SPSK system with a single polarization transmit antenna, the SPSK(DP) system proposed, with dual polarized transmit antennas, increases the spectral efficiency twice compared to the earlier system. The receiver combines the signal received from $N_{r}$ receive antennas. ML detector estimates the polarization index, the antenna index and the estimated symbol $\hat{s}$ is obtained.

\section{SPSK(DP) Performance Analysis}

The horizontal polarized component $E_{x}$ and vertical polarized component $E_{y}$ are represented as:

$$
\begin{aligned}
& E_{x}=a_{1} \cos \left(\omega t+\theta_{1}\right) \\
& E_{y}=a_{2} \cos \left(\omega t+\theta_{2}\right)
\end{aligned},
$$

where $a_{1}$ and $a_{2}$ are Rayleigh distributed random variables and $\theta_{1}$ and $\theta_{2}$ are uniformly distributed random variables. The signal received at the two antenna elements is given by:

$$
\begin{gathered}
V_{1}=E_{y} \cos \alpha+E_{x} \sin \alpha \\
V_{2}=-E_{x} \cos \alpha+E_{y} \sin \alpha
\end{gathered},
$$

where only the vertical component is present for $\alpha=0^{\circ}$ and only the horizontal component is present for for $\alpha=90^{\circ}$.

The dual polarized antenna at the receiver side, receives the transmitted signal with two branches. Due to the multipath effect, the signal with either horizontal or vertical polarization may get depolarized. So, the channel is assumed to be constant during the symbol time. The perfect knowledge of the channel state at the receiver side is assumed. Maximum Likelihood (ML) detection is performed at the receiver side. From the combined receive signal $Y$, first antenna index $\hat{j}$ is estimated, then the polarization index $\hat{p}$ of the active antenna is estimated based on cross-correlation comparing the signal strength from co-polar and cross-polar branches. The two estimation processes are interdependent. The average received power on the co-polar branches is greater than on the cross-polar branches. Therefore, polarization index estimation does not lead to errors. Hence, in the system with $N_{r}$ receive and $N_{t}$ transmit antennas the overall probability of error is reduced compared to SSK.

The signal at the receiver is:

$$
\left[\begin{array}{c}
y_{1} \\
y_{2} \\
\cdot \\
\cdot \\
\cdot \\
y_{N_{r}}
\end{array}\right]=\sqrt{E_{S}}\left[\begin{array}{c}
a_{k, 1} \mathrm{e}^{-j \theta_{k, 1}} \\
a_{k, 2} \mathrm{e}^{-j \theta_{k, 2}} \\
\cdot \\
\cdot \\
\cdot \\
a_{k, N_{r}} \mathrm{e}^{-j \theta_{k, N_{r}}}
\end{array}\right]+\left[\begin{array}{c}
n_{1} \\
n_{2} \\
\cdot \\
\cdot \\
\cdot \\
n_{N_{r}}
\end{array}\right] .
$$

The received signal in vector form is,

$$
Y=S_{k}+N \text {. }
$$

In Eq. (4), the received signal is represented as $\left[y_{1}, y_{2}, \ldots, y_{N_{r}}\right]^{T}, \sqrt{E_{s}}$ is the signal power, $a_{k, j}$ is amplitude and $\theta_{k, j}$ is angle of signal $s_{k}$ multiplied with channel coefficients $h_{j}$. $\left[n_{1}, n_{2}, \ldots, n_{N_{r}}\right]^{T}$ is the noise vector with AWGN symmetric parameters.

The decision metric $D_{k}$ is given by:

$$
\begin{gathered}
D_{i \mid u_{k}}=<y, \hat{s_{i}}>-\frac{1}{2}<\hat{s_{i}}, \hat{s_{i}}> \\
D_{i \mid u_{k}}=<\hat{s_{k}}, \hat{s_{i}}>+<w, \hat{s_{i}}>-\frac{1}{2}\left\|\hat{s_{i}}\right\|^{2}
\end{gathered} .
$$

The ML decision rule of SPSK(DP) for BPSK modulation is written as:

$$
\hat{u}=\left\{\begin{array}{ll}
u_{1} & \text { if } D_{1}>D_{2} \\
u_{2} & \text { otherwise }
\end{array},\right.
$$


where $<\hat{s}_{i}, \hat{s}_{i}>$ is the inner product of vectors $\hat{s}_{i}$ and $\hat{s}_{i}$. $u_{1}, u_{2}$ are binary symbols transmitted by signals $s_{1}(t)$ and $s_{2}(t)$.

The conditional error probability is given by [4]:

$$
\begin{gathered}
p(e \mid H)=\frac{1}{2} p_{u_{1}}(e \mid H)+\frac{1}{2} p_{u_{2}}(e \mid H), \\
p(e \mid H)=p\left(\left\|\hat{y}-\hat{s_{1}}\right\|^{2}-\left\|\hat{y}-\hat{s_{2}}\right\|^{2}\right), \\
p(e \mid H)=p\left(X>\left\|\hat{s_{1}}-\hat{s_{2}}\right\|^{2}\right), \\
p(e \mid H)=Q\left(\sqrt{\frac{E_{s}|| \hat{s_{1}}-\hat{s_{2}} \|^{2}}{4 N_{0}}}\right) .
\end{gathered}
$$

By the explanation of $Q$ function

$$
\begin{gathered}
p(e \mid H)=\frac{1}{\pi} \int_{0}^{\infty} \exp \left(\frac{\rho}{4 \sin ^{2} \theta}\right)^{N_{r}} f_{\rho}(\rho) \mathrm{d} \rho \mathrm{d} \theta \\
p(e \mid H)=\left.\mathscr{M}_{\rho}(s)^{N_{r}}\right|_{s=\frac{\rho}{4 \sin ^{2} \theta}} \mathrm{d} \theta .
\end{gathered}
$$

The Eq. 12 is reduced as closed form expression using [5]:

$$
p(e \mid H)=\left(\frac{1-\mu(c)}{2}\right) \sum_{k=0}^{N_{r}}\left(\frac{m-1+k}{k}\right)\left(\frac{1+\mu(c)}{2}\right)^{k},
$$

where $c=\frac{\rho}{4}$ and $\mu(c)=\sqrt{\frac{c}{1+c}}$.

The average error probability of $N_{t}$ transmit antennas is [4]:

$$
P_{b}=\frac{\frac{N_{t}}{2}}{N_{t}-1}\left[\frac{1}{N_{t}} \sum_{m=1}^{N_{t}} \sum_{m \neq n=1}^{N_{t}} p(e \mid H)\right] .
$$

\section{Results and Discussion}

For $N_{t}=4$ and $N_{r}=4$ transmit antennas SPSK symbol with the speed of $3 \mathrm{bits} / \mathrm{s} / \mathrm{Hz}$ is analyzed in the SPSK(DP) modulation scheme. The dual polarized antenna at the receiver side, receives the transmitted signal with two branches. The theoretical expressions are validated by Monte Carlo simulation. From Fig. 2, the SPSK system with dual polarized antennas SPSK(DP) outperforms both SSK and SPSK with a single polarized antenna. The power allocated to SPSK(SP) and SPSK(DP) is the same in the rich scattering Rayleigh environment. For the SNR of $14 \mathrm{~dB}$, SSK, SPSK(SP) and SPSK(DP) achieve the error performance of $1.48 \cdot 10^{-5}, 4.9 \cdot 10^{-6}$ and $3.57 \cdot 10^{-7}$, respectively. In the case of LOS, single polarized antennas will outperform dual polarized transmit antennas [4]. For the fixed average bit error rate (ABER) value, SPSK(DP) outperforms SSK and SPSK(SP) by $4.2 \mathrm{~dB}$ and $3 \mathrm{~dB}$, respectively.

From Fig. 3, the capacity of SPSK schemes is increased compared to SSK. In the SSK system, the information bits are transmitted only through the antenna index, and in SPSK, orthogonal polarization is also indexed for the transmission of information.

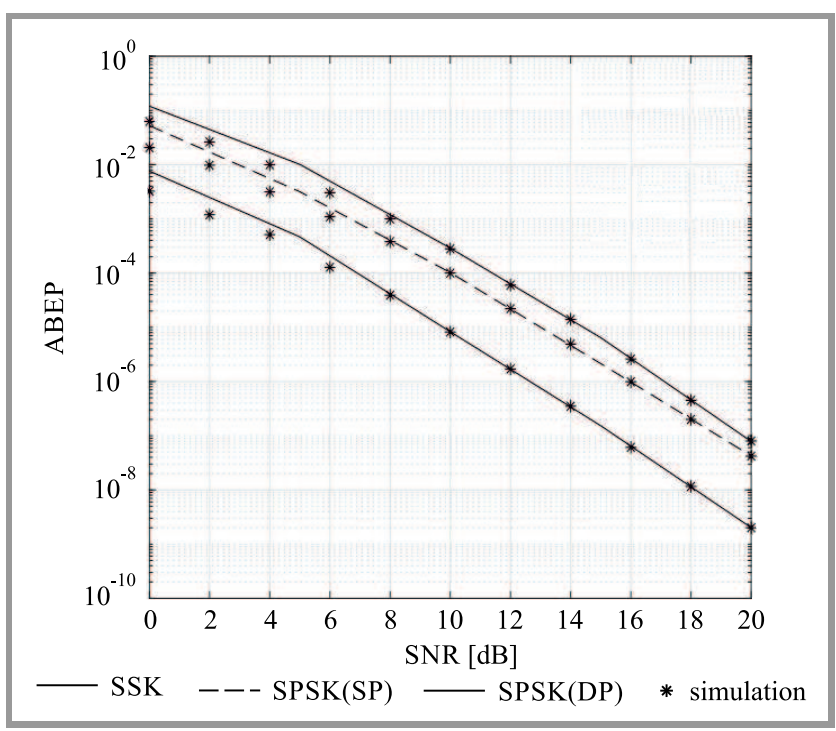

Fig. 2. Average bit error probability of different configurations compared to SSK.

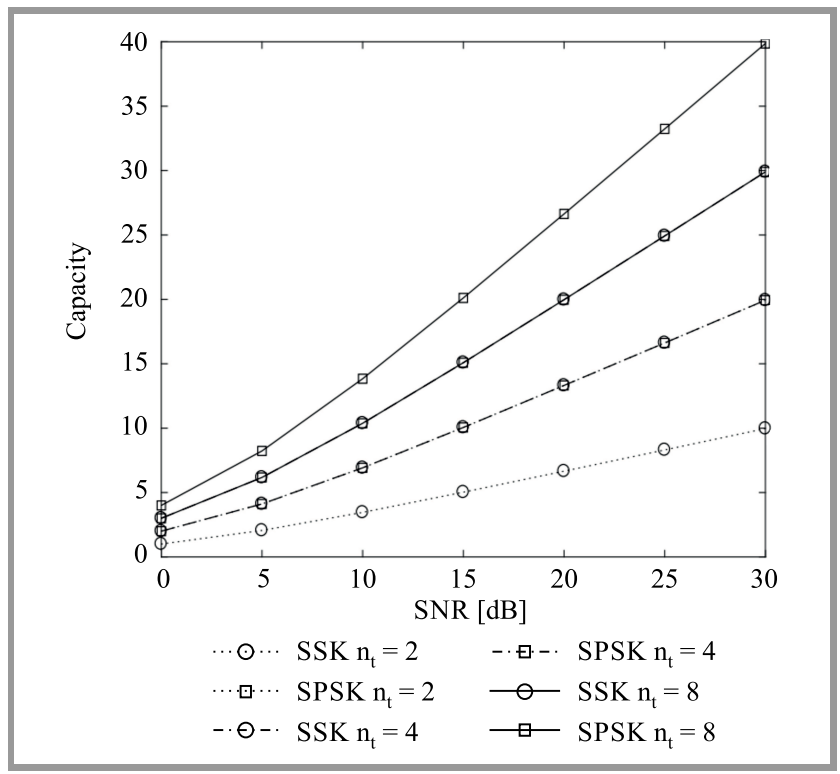

Fig. 3. Channel capacity of different configurations compared to SSK.

\section{Conclusions}

This paper compares the performance of SSK, SPSK(SP), SPSK(DP) systems. Dual polarized antennas at the transmitter and receiver of SPSK increase channel capacity and reduce error probability compared to SSK systems in the rich scattering environment. The increase in size of spatial constellation with dual polarized antennas results in better performance.

System performance studied over generalized fading channels is the subject of future work. The SPSK(DP) system can be experimentally evaluated using the NI LabVIEW software and the 18-slot PXIe chassis hardware platform. USRP RIO 2943R with the $2 \times 2$ MIMO configuration can be used as transceivers. 


\section{References}

[1] R. Mesleh and H. Haas, "Spatial modulation", IEEE Trans. on Veh. Technol., vol. 57, no. 4, pp. 2228-2241, 2008.

[2] M. Di Renzo and H. Haas, "A general framework for performance analysis of space shift keying (SSK) modulation for MISO correlated Nakagami-m fading channels", IEEE Trans. on Commun., vol. 58, no. 4, pp. 2590-2603, 2010.

[3] A. Kaur and G. Kaur, "Polarization shift keying modulation: A novel modulation technique for FSO systems", Int. J. of Engin. Develop. and Res., vol. 4, no. 2, pp. 1454-1459, 2016.

[4] S. Dhanasekaran, "Space-polarization shift keying modulation for MIMO channels", Wirel. Personal Commun., vol. 86, no. 3, pp. 1509-1539, 2016.

[5] M. K. Simon and M. S. Alouni, Digital Communication over Fading Channels: A Unified Approach to Performance Analysis, 1st ed. Wiley-Interscience, 2000.

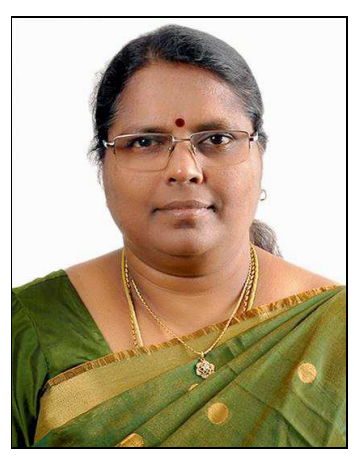

Malarvizhi Subramani is working as a Professor in the ECE Department, SRM University, Kattankulathur, Chennai, India. She finished her $\mathrm{Ph} . \mathrm{D}$. at the Wireless Communication College of Engineering, Guindy, Chennai. Her research interests are in the area of wireless communications, wireless sensor networks, MIMO and massive MIMO communications, as well as implementation of communications algorithms in FPGA.

E-mail: malarvizhi.g@ktr.srmuniv.ac.in

Department of Electronics and Communications

Engineering (ECE)

SRM IST-Kattankulathur

Chennai, India

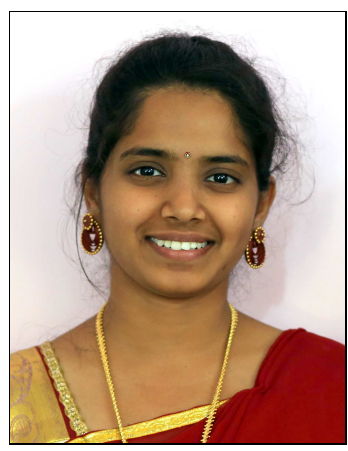

Arumbu Vanmathi Neduncheran is currently a research scholar in the ECE Department, SRM University, Kattankulathur, Chennai, India. She completed her M.Eng. in Communication Systems at the College of Engineering, Guindy, Chennai. She is a life member of ISTE. Her research interests are spatial modulation, cooperative communication, and massive MIMO.

E-mail: arumbu.n@ktr.srmuniv.ac.in

Department of Electronics and Communications

Engineering (ECE)

SRM IST-Kattankulathur

Chennai, India

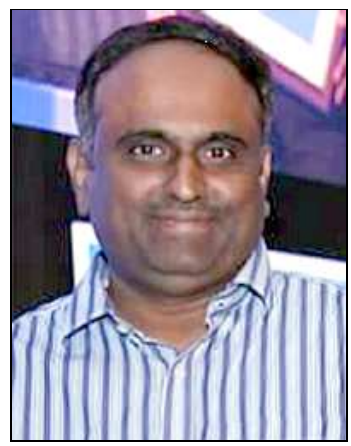

Vijayakumar Ponnusamy is working as an Assistant Professor in the ECE Department, SRM University, Chennai, India. He has completed his M.Eng. in Applied Electronics at the College of Engineering, Guindy, Chennai. He has completed his B.Eng. in Electronics and Communication Engineering from the Madras University. $\mathrm{He}$ is a member of the Communication Society of IEEE. His current research interests are in the area of MIMO wireless communications, cognitive radio networks, software defined radio and intelligent systems.

E-mail: vijayakumar.p@ktr.srmuniv.ac.in

Department of Electronics and Communications

Engineering (ECE)

SRM IST-Kattankulathur

Chennai, India 